Agronomía Costarricense 38(2): 33-41. ISSN:0377-9424 / 2014

www.mag.go.cr/rev_agr/index.html www.cia.ucr.ac.cr

\title{
PREVALENCIA DE HIPOCALCEMIA EN CUATRO HATOS JERSEY EN PASTOREO EN COSTA RICA
}

\author{
Jorge Ml. Sánchez ${ }^{1 / *}$, Alejandro Saborío-Montero* \\ Palabras clave: Hipocalcemia subclínica; hipocalcemia clínica; fiebre de leche; calificación condición corporal. \\ Keywords: Subclinical hypocalcemia; clinical hypocalcemia; milk fever; body condition score.
}

Recibido: 26/11/13

\section{RESUMEN}

La hipocalcemia clínica (HC) es una enfermedad metabólica común en vacas que pastorean forrajes suculentos altos en potasio. El objetivo de este estudio fue determinar la prevalencia de HC e hipocalcemia subclínica (HS) en 4 hatos Jersey en pastoreo en Costa Rica. Este último tipo de hipocalcemia ha sido poco estudiado en los sistemas de producción tropicales. Doscientos siete vacas Jersey de 4 hatos ubicados en las tierras altas de Cartago, Costa Rica, fueron utilizadas para analizar el contenido de calcio sérico $(\mathrm{Ca})$. La alimentación de los animales durante las 3 semanas previas al parto consistió en el pastoreo intensivo de pasto kikuyo (Kikuyuocloa clandestina) y la suplementación con 3 a 4 kilogramos de un alimento balanceado bajo en calcio y con $0,2 \%$ de la materia seca. Las muestras fueron ordenadas por número de lactancia: $1^{\circ}$ $(\mathrm{n}=36), 2^{\circ}(\mathrm{n}=48), 3^{\circ}(\mathrm{n}=49), 4^{\circ}(\mathrm{n}=41), 5^{\circ}(\mathrm{n}=17)$ y $6^{\circ}(\mathrm{n}=16)$. La concentración media de Ca sérico dentro del periodo crítico de incidencia de hipocalcemia ( $24 \mathrm{~h}$ previas o posteriores al parto) disminuyó con el número de lactancia. La incidencia de $\mathrm{HC}$ fue de 6, 13, 2, 29, 29 y $25 \%$ de las vacas de primera a sexta lactancia, respectivamente. Del mismo modo, la prevalencia de HS fue de

1 Autor para correspondencia. Correo electrónico: jorge.sanchezgonzalez@ucr.ac.cr
Aceptado: 29/05/14 
$53,42,78,44,47$ y $63 \%$, respectivamente, en el mismo orden. Las vacas con condición corporal (CC) de 3,75 o más en el parto \pm 1 semana tuvieron menor $(\mathrm{p}<0,05)$ concentración de calcio sérico que aquellas con $\mathrm{CC}$ inferiores; los contenidos de magnesio sérico fueron normales.

Los resultados muestran que la prevalencia de HS es mayor a $40 \%$ en hatos Jersey que consumen pastos tropicales; así mismo, sugieren que la CC durante el período de transición podría influir el contenido Ca sérico, sin embargo, debe hacerse más investigación para sustentar estos resultados.

\section{INTRODUCCIÓN}

La hipocalcemia clínica o fiebre de leche es un desbalance metabólico que afecta entre el 3 y $10 \%$ de las vacas durante el período comprendido entre las $24 \mathrm{~h}$ previas al parto y las 24 posteriores al mismo. La prevalencia de este desbalance metabólico está asociado a la raza, edad y composición mineral de la dieta que consume la vaca, siendo mayor en las denominadas razas del 'Canal', (Jersey y Guernsey) en los animales con 3 o más partos y en aquellos cuya alimentación se basa en el pastoreo intensivo de forrajes suculentos con contenidos altos de potasio (Goff 2008, Australia, State Government of Victoria 2007, Sánchez y Goff 2006, Roche 2003).

El periparto es un período de cambios abruptos en el metabolismo del Ca de la vaca lechera, ya que durante este período el animal pasa de un estado de gestación en que requiere alrededor de $12 \mathrm{~g}$ de Ca disponible por día $(\sim 10 \mathrm{~g}$ para mantenimiento $\mathrm{y} \sim 2 \mathrm{~g}$ para el desarrollo del feto), a un estado en que requiere cantidades muy altas de calcio disponible para sintetizar primero calostro esto es 1,7 a 2,3 g..$^{-1}$ y luego leche 1,1 g. $l^{-1}$ (NRC 2001). Para satisfacer la demanda de Ca para la producción la glándula mamaria tiene que remover entre 20 y $30 \mathrm{~g}$ de Ca por día de su plasma sanguíneo, el cual a su vez proviene de los líquidos extracelulares y de los canalículos del a body condition score (BCS) of 3.75 or higher at parturition \pm 1 week had lower $(\mathrm{p}<0.05)$ serum $\mathrm{Ca}$ concentration than those with lower BCS. Serum magnesium contents were normal. These results show that prevalence of $\mathrm{SH}$ is greater than $40 \%$ in Jersey herds grazing lush tropical pastures; also, data suggest that BCS during the transition period could influence serum $\mathrm{Ca}$ contents. However, more research should be done to support these findings.

hueso. Pese a que los niveles de $\mathrm{Ca}$ en el organismo están regulados por un sistema muy sensible que está orquestado por la hormona paratiroidea (HPT) y la $1,25(\mathrm{OH})_{2} \mathrm{D}_{3}$, presenta un desafío metabólico que hace que todas las vacas sufran algún grado de hipocalcemia durante el periparto. En la mayoría de los animales el sistema homeostático es capaz de compensar rápidamente la extracción de Ca que hace la glándula mamaria para producir calostro. En estos casos los niveles de Ca sanguíneo caen levemente y la vaca logra la normocalcemia en poco tiempo; en estos casos no hay consecuencias sobre la salud del animal. Así mismo, existen situaciones en las que el animal no logra compensar esa caída en los niveles de Ca sanguíneo durante el periparto, produciendo una reducción moderada en los niveles de este mineral que se denomina hipocalcemia subclínica. Cuando el animal sufre esta condición no presenta síntomas clínicos, sin embargo su productividad se puede afectar ya que el consumo de materia seca y la producción de leche se reducen durante el posparto. Si la caída de la concentración de Ca sanguíneo sobrepasa la capacidad del sistema homeostático para mantener la normocalcemia, la vaca sufre una hipocalcemia clínica o fiebre de leche que se caracteriza porque el animal pierde el tono muscular, tiene temblores musculares y finalmente cae. Esta 
condición puede predisponer al animal a sufrir enfermedades tanto metabólicas como infecciosas, ya que compromete el sistema inmune. Aunque las muertes por hipocalcemia clínica no son numerosas, fallece alrededor de un $5 \%$ de los animales que sufren el desbalance, por lo general los animales que mueren son los que producen más leche (Horst 1984, Horst et ál. 1994, Sánchez y Goff 2006, Goff 2006, Goff y Horst 1997, NRC 2001, Australia, State Government of Victoria 2007, Roche y Berry 2006).

Investigadores del Centro Nacional de Enfermedades de los Animales de los Estados Unidos (NADC, ARS, USDA, Ames, Iowa) han develado la magnitud de la prevalencia de hipocalcemia subclínica en hatos de ganado lechero confinados, reportando que más del $40 \%$ en las vacas multíparas sufren de este desbalance metabólico (Reinhardt et ál. 2011). Este grupo también ha explicado el rol que juega la hipocalcemia en la supresión del sistema inmune que experimentan las vacas durante el período de transición (Kimura et ál. 2006), lo que confirma las implicaciones que esta enfermedad tiene en la salud y por consiguiente sobre la productividad del hato de ganado lechero.

En ambientes tropicales, donde las vacas consumen pastos con contenidos altos en potasio (más de $3 \%$ de la materia seca), se ha reducido significativamente la prevalencia de hipocalcemia clínica al parto utilizando dietas con balances de calcio disponible ligeramente negativos durante las 2 o 3 semanas previas a la fecha prevista del parto (alrededor de 9 a $12 \mathrm{~g}$ de calcio disponible por vaca por día). Esta práctica de alimentación estimula el metabolismo del $\mathrm{Ca}$ al promover una ligera reducción de los contenidos de Ca sanguíneo que estimula la secreción de la hormona paratiroidea, la cual a su vez produce resorción osteoclástica del hueso y la síntesis de la hormona $1,25(\mathrm{OH})_{2} \mathrm{D}_{3}$. Esta última hormona promueve la absorción del $\mathrm{Ca}$ a nivel intestinal, incrementando los niveles de Ca plasmático. Una vez que la vaca pare y recibe la dieta de lactancia con cantidades altas de $\mathrm{Ca}$, el sistema homeostático es capaz de mantener la normocalcemia en momentos de alta demanda de este mineral. $\mathrm{La}$ homeostasis del $\mathrm{Ca}$ requiere de la presencia de $\mathrm{Mg}$, cuya disponibilidad puede ser reducida por la presencia de potasio en la dieta (Sánchez y Goff 2006, Goff 2006, Goff et ál. 2005).

El objetivo de esta investigación fue determinar la prevalencia de hipocalcemia clínica y subclínica en 4 hatos de ganado Jersey que consumen forrajes tropicales suculentos y con altos contenidos de potasio durante el periparto, a fin de develar la magnitud de la prevalencia de este desbalance metabólico y promover la utilización de prácticas de alimentación y manejo animal durante el período de vaca próxima al parto que mejoren la salud y productividad del hato de ganado lechero.

\section{MATERIALES Y MÉTODOS}

Durante el período comprendido entre enero y setiembre de 2012 se muestreó un total de 207 vacas Jersey de 4 hatos ubicados en Oreamuno, Cartago, Costa Rica ( $9^{\circ} 55^{\prime}$ Latitud Norte, $83^{\circ} 51^{\prime}$ Longitud Oeste, 2100 a $2400 \mathrm{msnm}$ ). La alimentación de los animales se basó en el uso intensivo del pasto kikuyo (Kikuyuocloa clandestina), cuyo valor nutricional promedio fue de $22 \%$ de PC, 1,35 Mcal de $\mathrm{EN}_{\mathrm{L}}$ (3X), 0,45\% de $\mathrm{Ca}, 0,40 \%$ de $\mathrm{P}, 0,32 \%$ de $\mathrm{Mg}, 3,87 \%$ de $\mathrm{K}$, $0,02 \% \mathrm{Na}, 0,70 \%$ de $\mathrm{Cl}, 0,22 \%$ de $\mathrm{S}$ y un BCAD de +665 meq. $\mathrm{kg}^{-1}$ de MS. Las 4 fincas cuentan con un grupo de vacas próximas al parto, donde los animales ingresan de 3 a 4 semanas antes de la fecha prevista de parto. Este grupo se suplementa con 3,5 kg por vaca por día de un alimento formulado para este estado fisiológico y cuya composición nutricional es $14 \%$ de PC, $1,72 \mathrm{Mcal}$ de $\mathrm{EN}_{\mathrm{L}} / \mathrm{kg}(3 \mathrm{X}), 0,25 \%$ de $\mathrm{Ca}, 0,40 \%$ de $\mathrm{P}, 0,42 \%$ de $\mathrm{Mg}, 1,38 \%$ de $\mathrm{K}, 0,03 \% \mathrm{Na}, 0,30 \%$ de $\mathrm{Cl}$, $0,18 \%$ de $\mathrm{S}$ y un BCAD de +170 meq. $\mathrm{kg}^{-1}$ de MS. Inmediatamente después del parto los animales fueron suplementados con alimentos balanceados y suplementos minerales acordes a sus requerimientos nutricionales.

Durante las $24 \mathrm{~h}$ previas o posteriores al parto se tomaron muestras de sangre de los 
vasos sanguíneos coccígeos utilizando agujas de 21" x 1" y tubos estériles de $9 \mathrm{ml}$ con activador de aglutinación de suero Z (Greiner Bio-One, tapa roja). Las muestras fueron identificadas y mantenidas a baja temperatura mientras se trasladaron al Centro de Investigaciones en Nutrición Animal (CINA) de la Universidad de Costa Rica, donde se centrifugaron por $15 \mathrm{~min}$ a $4^{\circ} \mathrm{C}, 3500$ rpm y separó el suero, el cual fue conservado en congelación. Los contenidos de $\mathrm{Ca}$ y $\mathrm{Mg}$ en las muestras de suero fueron determinados por espectrofotometría de absorción atómica (Zettner y Seligson 1964), utilizando un espectrofotómetro Perkin Elmer AAnalyst 800 equipado con un auto muestreador automático S10. Un umbral de Ca sérico de menos de 2,0 $\left(8 \mathrm{mg}^{-\mathrm{dl}^{-1}}\right)$ pero superior a $1,4\left(5,5 \mathrm{mg} . \mathrm{dl}^{-1}\right) \mathrm{mmol} . \mathrm{l}^{-1}$ se utilizó para definir las vacas como positivas para hipocalcemia subclínica y un valor inferior a 1,4 (5,5 mg.dl $\left.{ }^{-1}\right)$ mmol. $l^{-1}$ para hipocalcemia clínica. Así mismo, los contenidos de Magnesio $(\mathrm{Mg})$ en el suero inferiores a $0,75 \mathrm{mmol} . \mathrm{l}^{-1}\left(1,8 \mathrm{mg} . \mathrm{dl}^{-1}\right)$ fueron usados para definir hipomagnesemia, valores entre 0,75 a 1,0 mmol. $^{-1}\left(1,8\right.$ a $\left.2,4{\mathrm{mg} . \mathrm{dl}^{-1}}^{-1}\right)$ fueron considerados normales (Goff 2006). Las muestras se clasificaron por número de lactancia: $1^{\circ}$ $(\mathrm{n}=36), 2^{\circ}(\mathrm{n}=48), 3^{\circ}(\mathrm{n}=49), 4^{\circ}(\mathrm{n}=41), 5^{\circ}(\mathrm{n}=17)$ y $6^{\circ}(\mathrm{n}=16)$. Una persona calificó la condición corporal (CC, escala de 1 a 5 , propuesta por Ferguson et ál. 1994) semanalmente durante el peri parto (parto \pm 1 semana).

La información se analizó utilizando un análisis de varianza (ANOVA) para determinar las diferencias entre las variables continuas; así mismo se utilizó la prueba $t$ de Student para determinar las diferencias entre 2 variables continuas. La información fue analizada utilizando los paquetes sofware IBM SPSS Statistics $21.0^{\circledR} \mathrm{e}$ Infostat $1.0^{\circledR}$. La significancia estadística se declaró cuando $\mathrm{p}<0,05 \mathrm{y}$ el IC se determinó al 95\%.

\section{RESULTADOS Y DISCUSIÓN}

En la Figura 1 se presentan los valores de Ca sanguíneo de las vacas durante el período comprendido entre las $24 \mathrm{~h}$ previas y posteriores al parto, distribuidos según el número de parto y la condición de normocalcemia, hipocalcemia

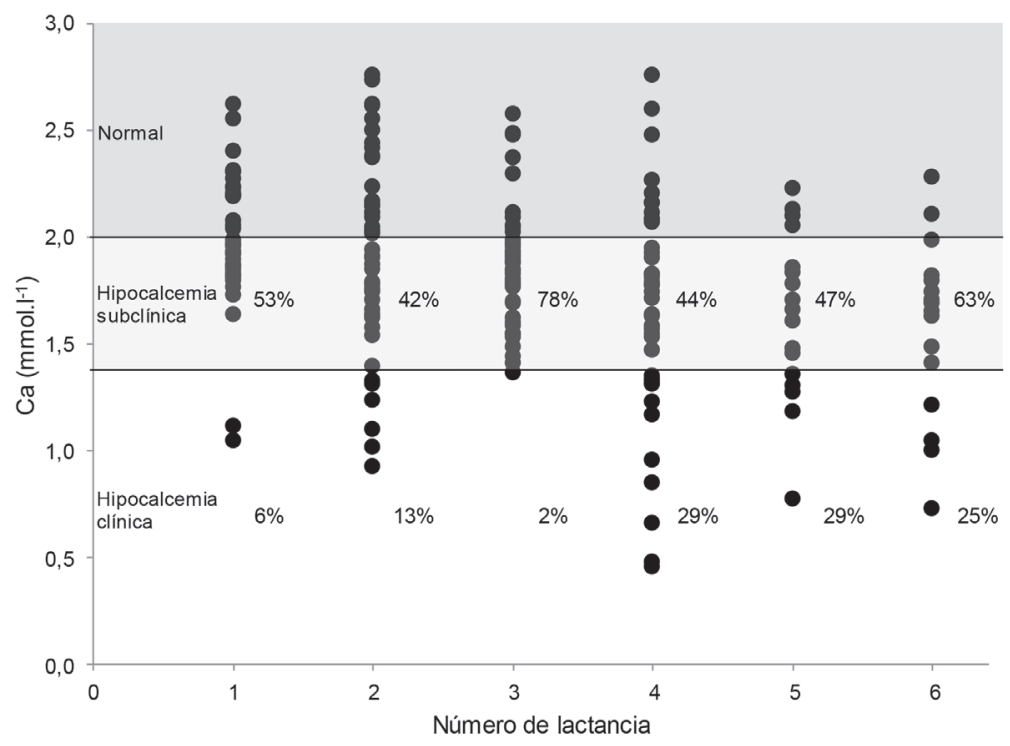

Fig. 1. Concentración de calcio en el suero sanguíneo durante el periodo de $24 \mathrm{~h}$ previas y posteriores al parto en 4 hatos de la raza Jersey en pastoreo en Cartago Costa Rica, graficada según el número de lactancia y la prevalencia de hipocalcemia clínica, subclínica o normocalcemia. 
subclínica e hipocalcemia clínica o fiebre de leche. La incidencia de hipocalcemia clínica; definida como la condición en que la concentración de Ca es menor a 1,4 mmol. $\mathrm{l}^{-1}\left(5,5 \mathrm{mg} . \mathrm{dl}^{-1}\right)$; fue mayor en los animales con un mayor número de partos, obteniéndose valores de 29, 29 y $25 \%$ en las vacas con 4, 5 o 6 partos. La prevalencia de hipocalcemia clínica en este estudio fue mayor a la observada por Reinhardt et ál. (2011) en un estudio realizado con animales estabulados, donde la raza predominante fue la Holstein. Estas diferencias pueden deberse a que el factor raza es determinante en la incidencia de hipocalcemia clínica, siendo las vacas Jersey más susceptibles que las Holstein a este desbalance metabólico (Goff et ál. 1995, NRC 2001). De los 21 animales correspondientes a esta subpoblación solamente 2 mostraron los síntomas propios de la fiebre de leche: animales caídos y con movimientos ruminales débiles y menos frecuentes.
El contenido de Ca sanguíneo disminuyó al aumentar el número de parto (Figura 2), encontrándose diferencias altamente significativas $(\mathrm{p}<0,01)$ entre los mismos. Así mismo, el contenido de Ca sanguíneo fue mayor $(\mathrm{p}<0,01)$ en los animales de primer parto que en los de 4 o más. Esta información es congruente con la literatura universal (NRC 2001), la cual indica que entre más partos tenga una vaca, el contenido de Ca sanguíneo al parto es menor y la probabilidad de sufrir fiebre de leche es mayor. Así mismo, estudios realizados por Horst et ál. (1990) han demostrado que cuando la vaca envejece, la cantidad de receptores hormonales de la 1,25 dihidroxivitamina $\mathrm{D}$ decrece a nivel intestinal y óseo, lo que reduce la capacidad del animal de absorber el Ca dietético y contribuir a reestablecer la normocalcemia inmediatamente después del parto.

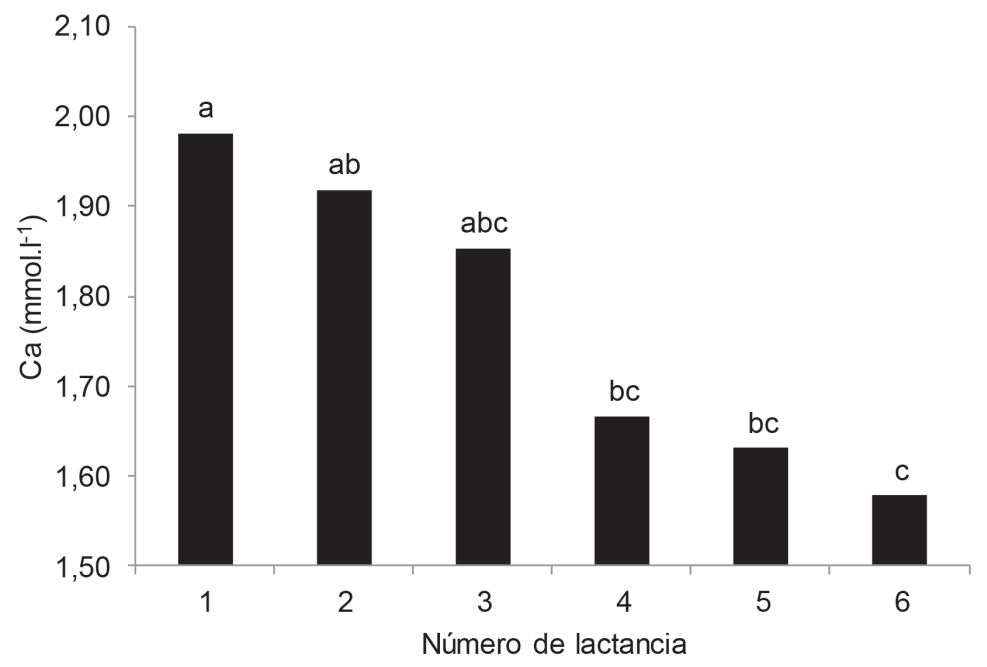

Fig. 2. Concentración promedio de calcio en suero sanguíneo dentro del periodo de 24 h antes o después del parto en vacas Jersey pastoreando forrajes tropicales de acuerdo con el número de lactancia. Columnas con letras distintas difieren $(\mathrm{p}<0,01)$ según la prueba de Tukey.

Curtis et ál. $(1983,1985)$ informan que la hipocalcemia clínica es una enfermedad económicamente muy importante para las fincas productoras de ganado lechero, ya que incrementa la susceptibilidad de las vacas a mastitis, retención de las membranas fetales, desplazamiento del abomaso, distocia y cetosis, lo que reduce la vida productiva de los animales y con ello los 
ingresos de los productores. Igualmente, Guard (1996) estima que las pérdidas por cada caso de hipocalcemia clínica es de $\$ 334$, en hatos confinados. Este valor es similar al encontrado por Sánchez 2014 (información no publicada) en sistemas pastoriles.

La prevalencia de hipocalcemia subclínica fue de $53,42,78,44,47$ y $63 \%$ para las vacas de 1 a 6 partos, respectivamente (Figura 1), lo que puede ser una manifestación de que las vacas requieren de horas; o tal vez de varios días; para que el intestino y el hueso responda a una mayor demanda fisiológica de $\mathrm{Ca}$ y el retorno a la normocalcemia (Goff y Horst 1997). En este estudio la prevalencia de hipocalcemia subclínica en vacas de primer parto fue mayor que la encontrada en hatos confinados (53 vs. $25 \%$ ), lo cual debe ser objeto de estudios específicos en animales en pastoreo, ya que según el NRC (2001) las vacas de primer parto tienen una gran capacidad de movilizar el Ca debido a que sus huesos aún están en crecimiento y además tienen una mayor cantidad de osteoclastos, lo que les permite responder más oportunamente a la demanda de $\mathrm{Ca}$ que se da durante el parto.

El grado de prevalencia de hipocalcemia subclínica en este estudio del segundo al sexto parto es alto y similar al encontrado en el estudio regional realizado por Reinhardt et ál. (2011) en los Estados Unidos, lo que puede tener implicaciones importantes en la salud y economía de los hatos de ganado lechero. Según la literatura (NRC 2001) esta reducción en los niveles de Ca circulante puede contribuir a reducir el apetito en las vacas recién paridas y predisponerlas a desarrollar enfermedades no infecciosas como cetosis y desplazamiento del abomaso, o bien enfermedades infecciosas como la retención de placenta y mastitis. Lo anterior debido a que según Kimura et ál. (2006) la reducción de los niveles de $\mathrm{Ca}$ sérico puede comprometer el sistema inmune.

La CC de las vacas fue evaluada durante los períodos de vaca próxima al parto y transición y se encontró que la concentración de $\mathrm{Ca}$ sérico fue significativamente $(\mathrm{p}<0,05)$ menor en las vacas con $\mathrm{CC}$ al parto ( \pm 1 semana) igual o mayor a 3,75 puntos, que aquellas con condición corporal menor (Figura 3). En promedio las vacas con CC igual o superior a 3,75 tuvieron concentraciones de Ca sérico de $1,72 \mathrm{mmol}^{-1}{ }^{-1}\left(6,8 \mathrm{mg} \mathrm{dl}^{-1}\right)$ al parto., lo que indica que estos animales son más propensos a sufrir hipocalcemia clínica y subclínica. Debido a lo anterior las prácticas de manejo y alimentación del hato de animales de la raza Jersey debe evitar el sobre acondicionamiento de las vacas para procurar la buena salud en las mismas.

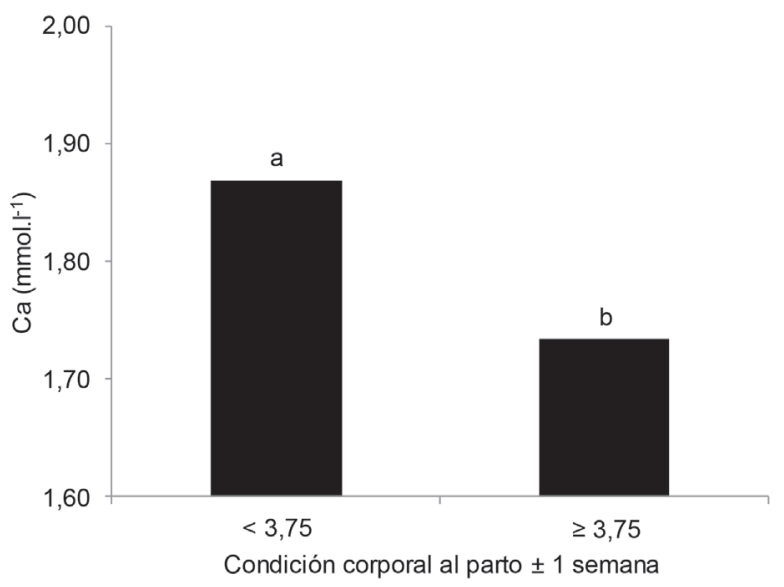

Fig. 3. Concentración media de calcio en suero sanguíneo dentro del periodo de $24 \mathrm{~h}$ antes o después del parto en vacas Jersey pastoreando forrajes tropicales de acuerdo con la CCC al parto \pm 1 semana. Columnas con letras distintas difieren $(\mathrm{p}<0,01)$ según la prueba de $\mathrm{T}$ de student para muestras independientes. 
El $\mathrm{Mg}$ es requerido para el metabolismo normal del $\mathrm{Ca}$, ya que es necesario para la secreción de la HPT y para que los receptores de la HPT sean sensibles a la misma (Goff 2006, Goff et ál. 2005). En sistemas pastoriles en que los forrajes manejados intensivamente son abundantes en potasio, el $\mathrm{Mg}$ es uno de los minerales más críticos para que una vaca pueda mantener su normocalcemia, ya que ambos minerales son antagónicos (Goff 2006, Sánchez y Goff 2006). En la Figura 4 se observa que únicamente 3 animales de los
207 estudiados tienen contenidos de $\mathrm{Mg}$ en la sangre inferiores a $0,75 \mathrm{mmol}^{-1}{ }^{-1}\left(1,8 \mathrm{mg} \cdot \mathrm{dl}^{-1}\right)$. Según van de Braak et ál. (1987) valores de $\mathrm{Mg}$ menores a 0,75 predisponen al animal a sufrir hipocalcemia, lo cual predispone al animal a sufrir fiebre de leche. Los resultados obtenidos en esta investigación sugieren que las vacas objeto de estudio recibieron una nutrición adecuada de Mg y no hay evidencias que sugieran que este mineral esté involucrado en la alta prevalencia de hipocalcemia clínica y subclínica observada en este estudio.

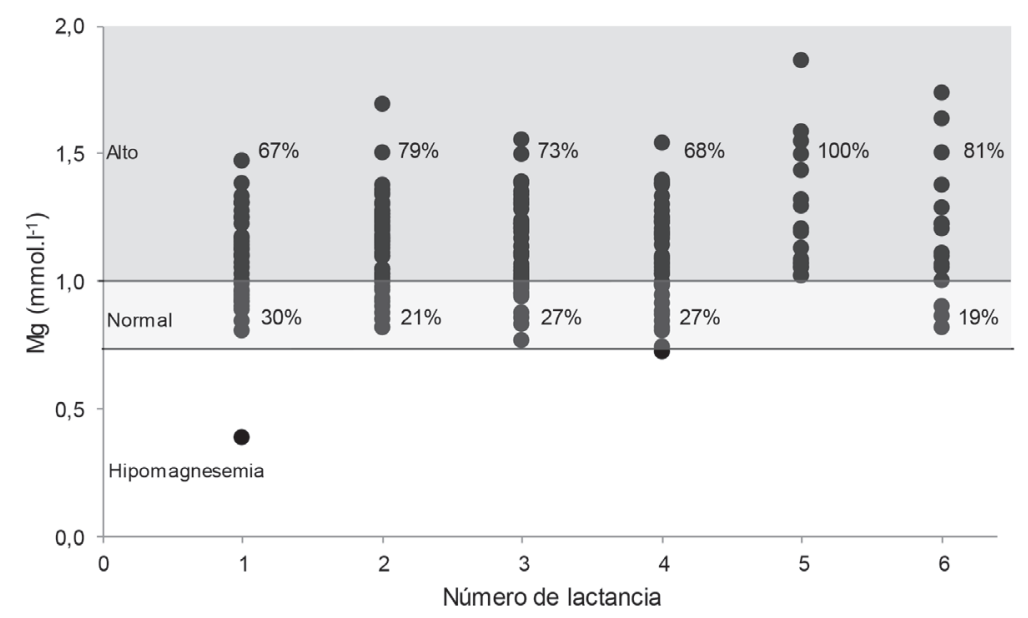

Fig. 4. Concentración de magnesio en el suero sanguíneo durante el periodo de 24 h previas y posteriores al parto en 4 hatos Jersey en pastoreo en Cartago Costa Rica, graficada según el número de lactancia y la prevalencia de valores considerados normales o altos.

Aunque en esta investigación el número de vacas caídas al parto fue muy baja, 2 de 207, la alta prevalencia de hipocalcemia clínica y subclínica observada sugiere la necesidad de seguir con el desarrollo de prácticas de manejo y alimentación tendientes a mejorar el metabolismo del Ca durante el período de transición de la vaca lechera. Lo anterior contribuirá a que la vaca logre la normocalcemia lo antes posible durante el período posparto, creando condiciones metabólicas para que la caída de los contenidos de Ca sanguíneo sea leve y de corta duración. Esta condición contribuirá a que la función neuromuscular y la respuesta inmune no se comprometan y a evitar la incidencia de enfermedades infecciosas y no infecciosas, las cuales son comunes durante el período de transición.

La baja incidencia de vacas caídas durante el periparto en este estudio, sugiere que el suministro de dietas bajas en Ca esto es 10 a 12 g de Ca disponible por vaca por día, aproximadamente durante el período de vaca próxima al parto contribuyen a prevenir los casos severos de hipocalcemia. 


\section{AGRADECIMIENTOS}

Los autores expresan su agradecimiento a los propietarios de las fincas "Los Espinos", Sr. Oscar Martínez, "Hacienda Pasquí", Ing. Agr. José Joaquín Jiménez, "El Paso”, Sra. Rosa Fernández y "El Plantón” Sr. Julio Sancho y Sr. Álvaro Sancho por facilitar los hatos objeto de estudio, además a los empleados de las fincas por su oportuna colaboración. Así mismo, agradecen al programa CRIPAS de la Escuela de Medicina Veterinaria de la Universidad Nacional, Heredia por permitir el uso del software VAMPP Bovino 3.0.

\section{LITERATURA CITADA}

AUSTRALIA, STATE GOVERNMENT OF VICTORIA. DEPARTMENT OF ENVIONMENTAL AND PRIMARY INDUSTRIES. AGRICULTURE AND FOOD. 2007. Milk fever (Hypercalcemia) in cows. Consultado 30 de diciembre de 2013. Disponible en http://www.depi.vic.gov.au/agriculture-and-food/ pests-diseases-and-weeds/animal-diseases/beefand-dairy-cows/milk-fever-hypocalcaemia-in-cows

CURTIS C.R., ERB H.N., SNIFFEN C.J., SMITH R. D., KRONFELD D.S. 1985. Path analysis of dry period nutrition, postpartum metabolic and reproductive disorders, and mastitis in Holstein cows. J. Dairy Sci. 68:2347-2360.

CURTIS C.R., ERB H.N., SNIFFEN C.J., SMITH R.D., POWERS P.A., SMITH M.C., WHITE M.E., HILMAN R.B., PEARSON E.J. 1983. Association of parturient hypocalcemia with eight periparturient disorders in Holstein cows. JAVMA 183:559-591.

FERGUSON J.P., GALLIGAN D.T., THOMSEN N. 1994. Principal descriptors of body condition score in Holstein cows. J. Dairy Sci. 77:2695-2703.

GOFF J.P. 2006. Macromineral physiology and application to the feeding of the dairy cow for prevention of milk fever and other periparturient mineral disorders. Animal Feed Science and Technology 126:237-257.

GOFF J.P. 2008. The monitoring prevention and treatment of milk fever and subclinical hypocalcemia in dairy cows. Vet. J. 176:50-57.

GOFF J.P., HORST R.L. 1997. Physiological changes at parturition and their relationship to metabolic disorders. J. Dairy Sci. 80:1260-1268.
GOFF J.P., REINHARDT T., HORST R.L. 1995. Milk fever and dietary cation-anion balance effects on concentration of vitamin D receptor in tissue of periparturient dairy cows. J. Dairy Sci. 78:23882394.

GOFF J.P., SÁNCHEZ J.M., HORST R.L. 2005. Hypocalcemia biological effects and strategies for prevention. Mineral Nutrition Conference. University of Tennessee. USA. 6 p.

GUARD C. 1996. Fresh cow problems are costly: culling hurts the most. Proc. 1994 Annu. Conf. Vet. Cornell Univ. Ithaca, NY.100 p.

HORST R.L. 1984. Regulation of calcium and phosphorus homeostasis in the dairy cow. J. Dairy Sci. 69:604616.

HORST R.L., GOFF J.P., REINHARDT T.A. 1990. Advancing age results in reduction of intestinal and bone 1,25 dihydroxyvitamin $\mathrm{D}$ receptor. Endocrinology 126:1053-1067.

HORST R.L., GOFF J.P., REINHARDT T.A. 1994. Calcium and vitamin D metabolism in the dairy cow. J. Dairy Sci. 77:1936-1951.

HORST R.L., GOFF J.P., REINHARDT T.A., BUXTON D.R. 1997. Strategies for preventing milk fever in dairy cattle. J. Dairy Sci. 80:1269-1280.

KIMURA K., REINHARDT T.A., GOFF J.P. 2006. Parturition and hypocalcemia blunts calcium signals in immune cells of dairy cattle. J. of Dairy Sci. 89:2588-2595.

NATIONAL RESEARCH COUNCIL. 2001. Nutrient Requirements of Dairy Cattle. $7^{\text {th }}$ rev. ed. National Academy Press Washington, D.C. 381 p.

REINHARDT T.A., LIPOLIS J.D., MC.CLUSKEY B.J., GOFF J.P., HORST R.L. 2011. Prevalence of subclinical hypocalcemia in dairy herds. The Veterinary Journal 188:122-124.

ROCHE J.R. 2003. The incidence and control of hypocalcemia in pasture-based systems. Acta Vet. Scand. Suppl. 97:141-144.

ROCHE J.R., BERRY D.P. 2006. Periparturient climatic, animal, and management factors influencing the incidence of milk fever in grazing systems. J. Dairy Sci. 89:2775-2783.

SÁNCHEZ J.M., GOFF J.P. 2006. Strategies for controlling hypocalcemia in dairy cows in confinement and pastures, pp. 182-187. In: Production diseases in farm animals. Edited by N. Joshi and T.H. Herdt. Wageningen Academic Publishers. Wageningen, The Netherlands. 
VAN DE BRAAK A.E., VAN'T KLOOSTER A.T., MALESTEIN A. 1987. Influence of a deficient supply of magnesium during the dry period on the rate of calcium mobilization by dairy cows at parturition. Res. Vet. Sci. 42:101-108.
ZETTNER A., SELIGSON. D. 1964. Application of atomic absorption spectrophotometry in the determination of calcium in serum. Atomic Absorption Spectrophotometry 10(2):869-890.

(c) $\$($ Todos los derechos reservados. Universidad de Costa Rica. Este artículo se encuentra licenciado con Creative Commons Reconocimiento-NoComercial-SinObraDerivada 3.0 Costa Rica. Para mayor información escribir a rac.cia@ucr.ac.cr 
